\title{
Role of aldolase $A$ in osteosarcoma progression and metastasis: In vitro and in vivo evidence
}

\author{
FENG LONG ${ }^{1}, \mathrm{XINYAN} \mathrm{CAI}^{2}, \mathrm{WEI} \mathrm{LUO}^{1}, \mathrm{LIANG} \mathrm{CHEN}^{1}$ and $\mathrm{KANGHUA} \mathrm{LI}^{1}$ \\ Departments of ${ }^{1}$ Orthopaedics and ${ }^{2}$ Neonatology, Xiangya Hospital, \\ Central South University, Changsha, Hunan 410078, P.R. China
}

Received December 7, 2013; Accepted February 10, 2014

DOI: $10.3892 /$ or.2014.3473

\begin{abstract}
Aldolase A (ALDOA) has been reported to be negative survival marker of osteosarcoma (OS) and may be implicated in OS development and progression. In the present study, we assessed for the first time the functional role of ALDOA in OS cell invasion and survival in vitro and in vivo, using human OS cell lines and an orthotopic xenograft nude mouse model. Overexpression and knockdown of ALDOA were respectively performed in MG-63 and U-2 OS cells, which showed relatively low and high constitutive ALDOA expression levels, respectively. Overexpression of ALDOA in MG-63 cells significantly increased in vitro cell invasion, matrix metalloproteinase (MMP)-2 expression, and cell survival against cisplatin-induced apoptosis. On the other hand, knockdown of ALDOA in U-2 cells markedly decreased in vitro cell invasion, MMP-2 expression, and cell survival against cisplatin-induced apoptosis. In an orthotopic xenograft nude mouse model, intra-tibial injection of MG-63 cells overexpressing ALDOA led to significantly increased primary tumor volume and pulmonary metastasis as well as decreased cell apoptosis in the primary tumors, compared with the controls. In contrast, intra-tibial injection of U-2 cells with knockdown of ALDOA led to markedly decreased primary tumor volume and pulmonary metastasis as well as increased cell apoptosis in the primary tumors, compared with the controls. In conclusion, our in vitro data indicate that ALDOA promotes OS cell invasion and survival, and our in vivo data demonstrate an important role of ALDOA in promoting OS tumor growth and metastasis. The present study provides the first in vitro and in vivo evidence supporting a critical functional role of ALDOA in OS progression and metastasis, suggesting that ALDOA could serve as a novel therapeutic
\end{abstract}

Correspondence to: Dr Kanghua Li, Department of Orthopaedics, Xiangya Hospital, Central South University, 87 Xiangya Road, Changsha, Hunan 410078, P.R. China

E-mail:1kh8738@sina.com

Key words: aldolase A, osteosarcoma, metastasis, cell invasion, matrix metalloproteinase-2, cell survival, apoptosis, orthotopic xenograft target in OS. Additionally, our results suggest that ALDOA is involved in the development of OS chemoresistance.

\section{Introduction}

Osteosarcoma (OS) is the most common primary bone malignancy and the eighth most common type of cancer among children, comprising $2.4 \%$ of all malignancies in pediatric patients and $\sim 35 \%$ of all bone cancers (1). The overall incidence is five cases per million individuals per year (1). OS is a devastating disease, characterized by high local aggressiveness and a tendency to metastasize to the lungs and distant bones. Pulmonary metastasis occurs in $\sim 40-50 \%$ of OS patients and remains a major cause of fatal outcome (2-4). The cure rate of OS is $\sim 65 \%$ for patients with localized diseases. When presenting with metastases at the time of diagnosis, the survival rate is $25 \%(4,6)$. Despite modern multidisciplinary treatments including chemotherapy and surgery, the 5-year survival rate of osteosarcoma patients remains $60-70 \%$ (1). Hence, there is an urgent need to develop novel approaches to treat osteosarcoma patients, particularly, to identify and confirm potential therapeutic targets involved in OS development and progression.

A new approach to therapeutic strategy is emerging, based on the peculiar metabolism of the cancer cell. Specifically, glycolysis has long been considered the main source of energy for the cancer cell (7). Fructose-bisphosphate aldolase (EC 4.1.2.13) is involved in glycolysis by converting fructose 1,6-diphosphate into dihydroxyacetone phosphate and glyceraldehyde-3-phosphate (8). The three aldolase isozymes (A, $\mathrm{B}$ and $\mathrm{C}$ ) have a tetramer structure with identical molecular weights of $\sim 160 \mathrm{kDa}$. It is well known that cancer cells with a high glycolytic rate often exhibit an aberrant expression of all glycolytic enzymes (8). It has been found that the control of glycolysis in rapidly growing tumor cells occurs at least partly at the level of the so-called consuming block (from aldolase to lactate dehydrogenase) (9). Accumulation of fructose-1,6-bisphosphate resulting from inhibition of aldolase-catalyzed cleavage should stop glycolysis and, therefore, cancer development and progression (8).

A recent study revealed that the expression of aldolase $A$ (ALDOA) was significantly higher in OS patients with shorter survival time, suggesting that ALDOA is a negative survival marker of OS and may be implicated in OS development and 
progression (10). In the present study, for the first time we assessed the functional role of ALDOA in OS cell invasion and survival in vitro and in vivo, using human OS cell lines and an orthotopic xenograft OS nude mouse model.

\section{Materials and methods}

Cells lines, plasmids, reagents and mice. MG-63 and U-2 human OS cell lines were purchased from the American Type Culture Collection (ATCC; Rockville, MD, USA). Human $A L D O A$ cDNA was subcloned into the pcDNA 3.1 expression vector (11). ALDOA (sc-29664-V) shRNA lentiviral particles, control shRNA lentiviral particles-A (sc-108080), and anti-ALDOA (N-15) (sc-12059) and anti-matrix metalloproteinase-2 (MMP-2) antibodies (sc-53630) were purchased from Santa Cruz Biotechnology (Santa Cruz, CA, USA). The aldolase activity in cell extracts was analyzed with an aldolase test kit (CALD 015) purchased from Caldon Biotech (Vista, CA, USA). DeadEnd ${ }^{\mathrm{TM}}$ Fluorometric TUNEL system was purchased from Promega (Madison, WI, USA). ApopTag ${ }^{\circledR}$ Peroxidase In Situ Apoptosis Detection kit (S7100) was purchased from Millipore (Billerica, MA, USA). SuperFect ${ }^{\mathrm{TM}}$ transfection reagent was purchased from Qiagen (Valencia, CA, USA). Puromycin, G418, cisplatin and all chemicals of reagent grade were purchased from Sigma (St. Louis, MO, USA). Fiveweek-old BALB/C female nude mice were purchased from Central South University (Changsha, China) and were housed at the Xiangya Hospital BioResources Centre. All animal care, breeding and testing procedures were approved by the Laboratory Animal Users Committee of Xiangya Hospital, Central South University, Changsha, China.

Transfection and lentiviral transduction. The human ALDOA expression constructs were transfected into MG-63 cells using SuperFect ${ }^{\mathrm{TM}}$ transfection reagent, and pools of stable transductants were generated via selection with $\mathrm{G} 418(800 \mu \mathrm{g} / \mathrm{ml})$ according to the manufacturer's protocol (Qiagen). Lentiviral transduction was performed in U-2 cells, and pools of stable transductants were generated via selection with puromycin (5 $\mu \mathrm{g} / \mathrm{ml})$ according to the manufacturer's protocol (Santa Cruz Biotechnology).

In vitro cell invasion assay. Transwell ${ }^{\circledR}$ cell invasion assays (Corning Life Sciences, Tewksbury, MA, USA) were performed as previously described (12). Briefly, Transwell ${ }^{\circledR}$ cell-culture chambers with an $8-\mu \mathrm{m}$ pore size (BD Biosciences, Bedford, MA, USA) for 24-well plates were coated with $50 \mu 1$ Matrigel (BD Biosciences). OS cells were seeded into the upper chamber at $5 \times 10^{5}$ cells/well in RPMI-1640 serum-free medium. Complete medium $(600 \mathrm{ml})$ was added to the lower chamber. Cells were allowed to invade for $24 \mathrm{~h}$ followed by fixation and staining with crystal violet. Invaded cells which adhered to the bottom of the filter were counted in 10 random fields per chamber under a microscope. Each experiment was repeated three times in triplicates.

Western blot analysis. Immunoblotting was performed with the respective antibodies. Briefly, cells were dissolved in $250 \mu \mathrm{l}$ of $2 \mathrm{X}$ SDS loading buffer $(62.5 \mathrm{~mm}$ Tris- $\mathrm{HCl}$, pH 6.8, 2\% SDS, 25\% glycerol, $0.01 \%$ bromophenol blue, $5 \%$ 2-mercaptoethanol), and incubated at $95^{\circ} \mathrm{C}$ for $10 \mathrm{~min}$. Equal amounts of proteins for each sample were separated by $10 \%$ SDS-polyacrylamide gel and blotted onto a polyvinylidene difluoride microporous membrane (Millipore). Membranes were incubated for $1 \mathrm{~h}$ with a $1 / 1,000$ dilution of primary antibody, and then washed and revealed using secondary antibodies with horseradish peroxidase conjugate $(1 / 5,000$, $1 \mathrm{~h})$. Peroxidase was revealed with a GE Healthcare ECL kit. Proteins were quantified before being loaded onto the gel.

Measurement of apoptosis by terminal deoxynucleotidyl transferase mediated nick-end labeling (TUNEL) assay. The TUNEL assay was performed using the DeadEnd ${ }^{\mathrm{TM}}$ Fluorometric TUNEL system according to the manufacturer's protocol (Promega). Cells were treated with cisplatin $(10 \mathrm{nM})$ for $8 \mathrm{~h}$. Apoptotic cells exhibit a strong nuclear green fluorescence that can be detected using a standard fluorescein filter. All cells stained with DAPI exhibit a strong blue nuclear fluorescence. The slides were observed using fluorescence microscopy and the relative apoptotic cells were determined by counting TUNEL-positive cells in five random fields (magnification, x100) for each sample.

Establishment of an orthotopic xenograft OS nude mouse model. OS cells were mixed with 50\% Matrigel to a concentration of $2 \times 10^{6}$ cells $/ \mathrm{ml}$. Mice were anesthetized by intraperitoneal injection of ketamine $(100 \mathrm{mg} / \mathrm{kg}$ body weight) and xylazine (10 mg/kg body weight). A volume of $10 \mu \mathrm{l}$ of cells/Matrigel solution was injected into the left tibia of individual nude mice using a 27-gauge needle (12). The needle was inserted into the tibial tuberosity and advanced using a drilling motion to avoid fracture of the bone. The mice were monitored three times weekly for tumor growth and signs of distress. Tumors were measured in the anteroposterior and lateral planes using digital callipers. Leg volume and tumor volume were calculated using the formula: $4 / 3 \pi\left(1 / 4(\mathrm{AP}+\mathrm{L})^{2}\right.$ where AP is the anteroposterior measurement and $\mathrm{L}$ is the lateral measurement (13). The volume of the contralateral limb was subtracted from the tumor-bearing limb to calculate the actual tumor volume. Mice were weighed using digital scales. Tumor growth was evaluated until death or sacrifice when tumor dimensions exceeded $5 \%$ of the body weight or mice showed dyspnea, abnormal posture, $>20 \%$ body weight loss, difficulty with ambulation or any other clinical sign of metastatic disease causing significant pain or distress, according to the institutional guidelines.

Clonogenic lung metastasis assay. Clonogenic lung metastasis assays were performed as previously described $(14,15)$. Briefly, lungs from each individual animal were minced into $1-\mathrm{mm}$ pieces, and digested with $5 \mathrm{ml}$ enzyme cocktail containing $1 \mathrm{mg} / \mathrm{ml}$ collagenase IV and 6 units $/ \mathrm{ml}$ elastase in PBS for $1 \mathrm{~h}$ at $4{ }^{\circ} \mathrm{C}$ with rotation. Cell suspensions were filtered through 70-Amnylon cell strainers and washed two times with Hank's buffered saline, and then resuspended in complete medium. The cells were then cultured in $10-\mathrm{cm}$ tissue culture dishes and treated with $1.25 \mathrm{mg} / \mathrm{ml}$ of G418 or $5 \mu \mathrm{g} / \mathrm{ml}$ of puromycin to allow only the growth of MG-63 and U-2 cells, respectively. When colonies of the growing cells became visible (8-14 days), the plates were washed with phosphate-buffered saline, fixed with methanol and stained 
A

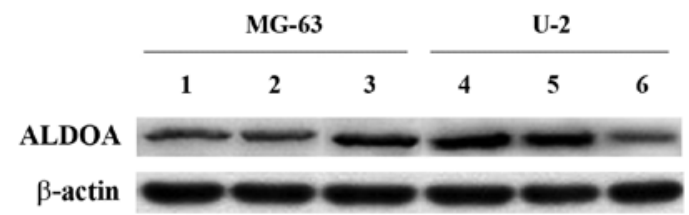

B

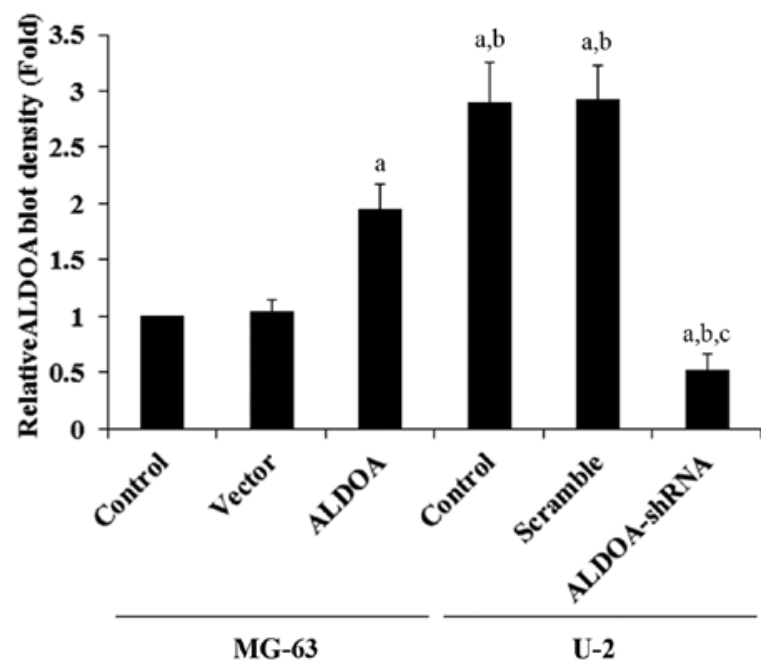

Figure 1. Aldolase A (ALDOA) expression in osteosarcoma cells with overexpression or knockdown of ALDOA. (A) In the MG-63 cells, expression of ALDOA in the control cells (control, lane 1), cells stably transfected with the empty pcDNA3 vector (vector, lane 2), and cells stably transfected with ALDOA (lane 3) was analyzed by western blot analysis. In the U-2 cells, expression of ALDOA in control cells (control, lane 4), cells stably transduced with scramble control shRNA (scramble, lane 5), and cells stably transduced with ALDOA-shRNA (lane 6 ) was analyzed by western blot analysis. $\beta$-actin blotting was used as a loading control. Protein blots were measured by densitometry. (B) Density of the ALDOA blot was normalized against that of $\beta$-actin to obtain a relative blot density, which was expressed as fold-change to the relative ALDOA blot density of MG-63 control cells (designated as 1). ${ }^{a} \mathrm{P}<0.05$ compared with (MG-63) control and vector; ${ }^{\mathrm{b}} \mathrm{P}<0.05$ compared with (MG-63) ALDOA; ${ }^{\circ} \mathrm{P}<0.05$ compared with (U-2) control and scramble.

with crystal violet. The colonies were counted independently by two investigators, blinded to the group to which each nude mouse belonged, and the total colony number/lungs was calculated for each animal.

Immunohistochemistry. Apoptosis in the primary OS tumor tissue was evaluated using the ApopTag ${ }^{\circledR}$ Peroxidase In Situ Apoptosis Detection kit according to the manufacturer's protocol (Millipore). Light hematoxylin was used for counterstaining. After staining, apoptosis was determined from 500 randomly selected cells as the proportion of cells with apoptotic nuclei.

Statistical analysis. Statistical analyses were performed with SPSS for Windows 10.0. Data values are expressed as means \pm SD. Comparison of the means between two independent groups was performed with the Student's t-test. Comparison of the means among multiple groups was performed with one-way ANOVA followed by post hoc pairwise comparisons using Tukey's tests. Two-tailed $\mathrm{P}<0.05$ was considered to indicate a statistically significant result in the present study.

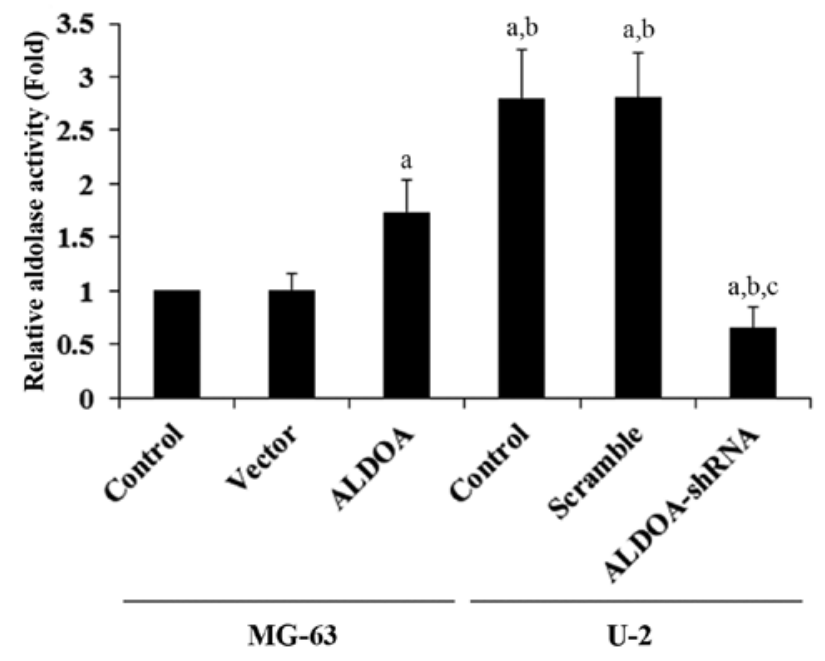

Figure 2. Aldolase activity in osteosarcoma cells with overexpression or knockdown of aldolase A (ALDOA). In MG-63 cells, the ALDOA activity was determined in cell extracts from control cells (control), cells stably transfected with empty pcDNA3 vector (vector), and cells stably transfected with ALDOA. In U-2 cells, the ALDOA activity was determined in cell extracts from control cells (control), cells stably transduced with scramble control shRNA (scramble), and cells stably transduced with ALDOA-shRNA. The aldolase activity was expressed as fold-change to that of the MG-63 control cells (designated as 1 ). ${ }^{\text {a }} \mathrm{P}<0.05$ compared with (MG-63) control and vector; ${ }^{\mathrm{b}} \mathrm{P}<0.05$ compared with (MG-63) ALDOA; ${ }^{\mathrm{c}} \mathrm{P}<0.05$ compared with (U-2) control and scramble.

\section{Results}

As shown in Fig. 1, while ALDOA was amply expressed in U-2 OS cells, it was expressed at a relatively low constitutive level in the MG-63 cells. The two cell lines allowed the specific ALDOA overexpression or knockdown studies to be performed in the context of the study goals. Thus, we stably transfected MG-63 cells with an ALDOA expression vector to overexpress ALDOA, and we stably transduced U-2 cells with ALDOA-shRNA to knock down ALDOA. Compared with the controls, ALDOA was overexpressed 2 -fold in the MG-63 cells, and the endogenous ALDOA level was knocked down $\sim 80 \%$ in the U-2 cells (Fig. 1). Aldolase activity assays using the cell extracts showed that U-2 cells had higher constitutive aldolase activity when compared with that in the MG-63 cells (Fig. 2). ALDOA overexpression significantly increased the aldolase activity in the MG-63 cells, when compared with the controls. On the other hand, ALDOA knockdown markedly decreased the aldolase activity in the U-2 cells (Fig. 2).

To examine the effect of ALDOA on OS cell invasion, we performed in vitro cell invasion assays and examined the MMP expression level in OS cells. Overexpression of ALDOA in MG-63 cells increased cell invasion by $\sim 1$-fold when compared with that of the controls, while knockdown of ALDOA in U-2 cells decreased cell invasion by $\sim 80 \%$ (Fig. 3). A similar trend in data was observed for the expression of MMP-2 (Fig. 4).

To explore the effect of ALDOA on OS survival, we examined cell apoptosis in OS cells treated with $10 \mathrm{nM}$ of cisplatin, an apoptosis-inducing chemotherapeutic agent commonly used to treat OS. Overexpression or knockdown of ALDOA did not significantly alter the rate of cell apoptosis in both 


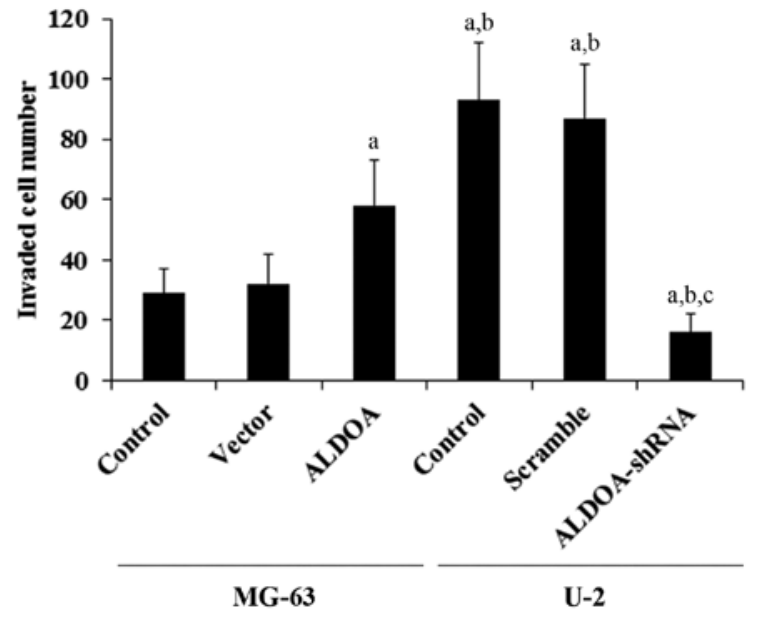

Figure 3. In vitro cell invasion in osteosarcoma cells with overexpression or knockdown of aldolase A (ALDOA). In MG-63 cells, in vitro cell invasion assays were performed in control cells (control), cells stably transfected with empty pcDNA3 vector (vector), and cells stably transfected with ALDOA. In U-2 cells, in vitro cell invasion assays were performed in control cells (control), cells stably transduced with scramble control shRNA (scramble), and cells stably transduced with ALDOA-shRNA. Invaded cell numbers were counted. ${ }^{\mathrm{a}} \mathrm{P}<0.05$ compared with (MG-63) control and vector; ${ }^{\mathrm{b}} \mathrm{P}<0.05$ compared with (MG-63) ALDOA; ${ }^{\mathrm{C}} \mathrm{P}<0.05$ compared with (U-2) control and scramble.

A

\begin{tabular}{ccccccc}
\multicolumn{3}{c}{ MG-63 } & & \multicolumn{3}{c}{ U-2 } \\
\cline { 1 - 3 } \cline { 5 - 7 } 1 & 2 & 3 & & 5 & 5 & 6
\end{tabular}

MMP-2

$\beta$-actin

B

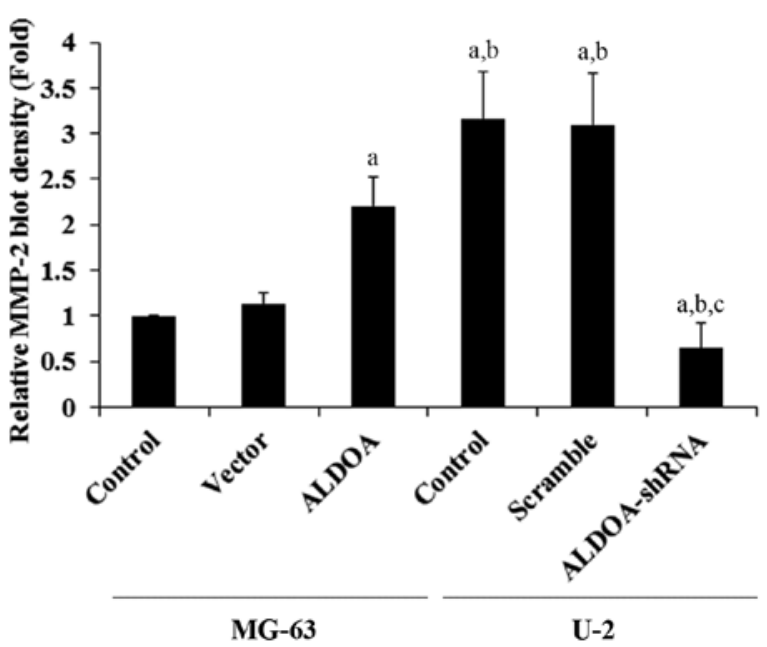

Figure 4. Matrix metalloproteinase-2 (MMP-2) expression in osteosarcoma cells with overexpression or knockdown of aldolase A (ALDOA). (A) In MG-63 cells, expression of MMP-2 in control cells (control, lane 1), cells stably transfected with empty pcDNA3 vector (vector, lane 2), and cells stably transfected with ALDOA (lane 3) was analyzed with western blot analysis. In U-2 cells, expression of MMP-2 in control cells (control, lane 4), cells stably transduced with scramble control shRNA (scramble, lane 5), and cells stably transduced with ALDOA-shRNA (lane 6) was analyzed with western blot analysis. $\beta$-actin blotting was used as a loading control. Protein blots were measured by densitometry. (B) Density of the MMP-2 blot was normalized against that of $\beta$-actin to obtain a relative blot density, which was expressed as fold-change to the relative MMP-2 blot density of MG-63 control cells (designated as 1). ${ }^{\mathrm{a}} \mathrm{P}<0.05$ compared with (MG-63) control and vector; ${ }^{\mathrm{b}} \mathrm{P}<0.05$ compared with (MG-63) ALDOA; ${ }^{\mathrm{P}} \mathrm{P}<0.05$ compared with (U-2) control and scramble.
A

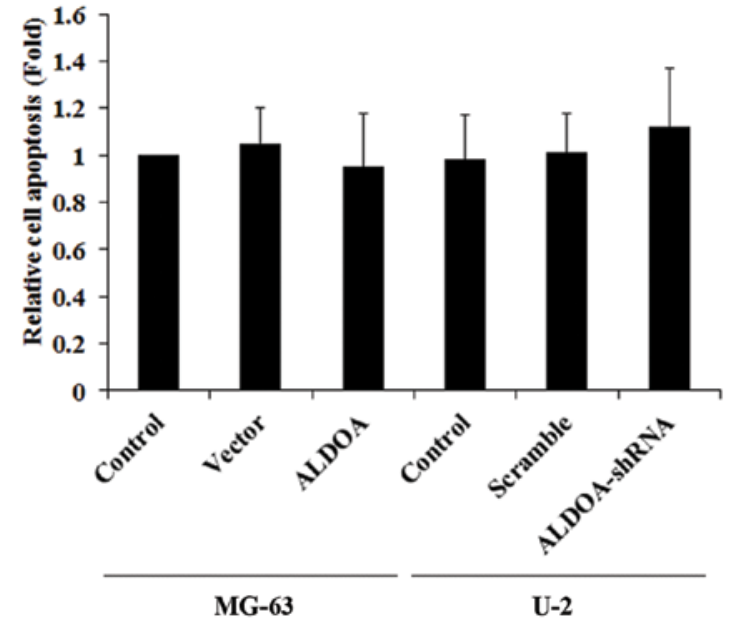

B

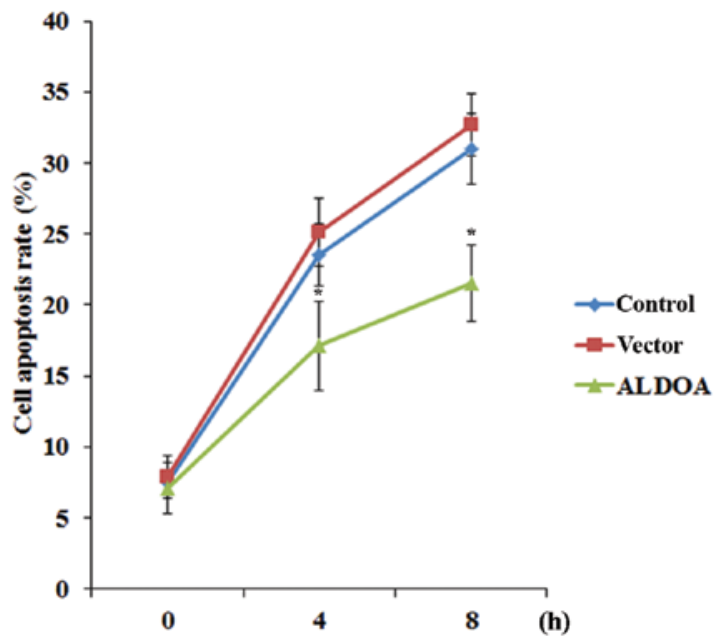

C

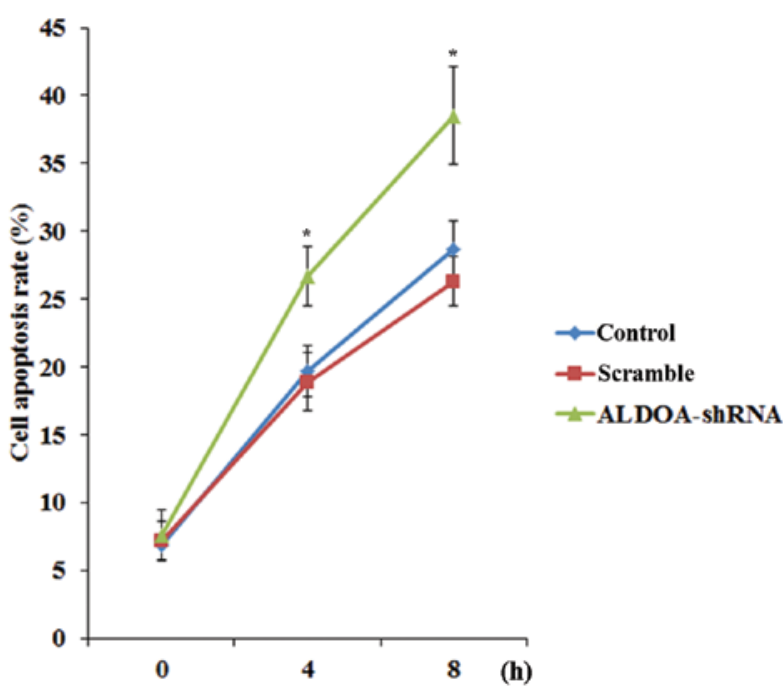

Figure 5. Cisplatin-induced apoptosis in osteosarcoma cells with overexpression or knockdown of aldolase A (ALDOA). In MG-63 cells, TUNEL (terminal deoxynucleotidyl transferase mediated nick-end labeling) assays were performed in control cells (control), cells stably transfected with empty pcDNA3 vector (vector), and cells stably transfected with ALDOA. In U-2 cells, TUNEL assays were performed in control cells (control), cells stably transduced with scramble control shRNA (scramble), and cells stably transduced with ALDOA-shRNA. (A) The cells were under normal culture conditions for $8 \mathrm{~h}$. The cell apoptosis rate at $8 \mathrm{~h}$ was expressed as fold-change to that of the MG-63 control cells (designated as 1). (B) MG-63 and (C) U-2 cells were treated with $10 \mathrm{nM}$ of cisplatin for $8 \mathrm{~h}$. The cell apoptosis rates at 4 and $8 \mathrm{~h}$ were shown as the percentage of TUNEL-positive cells among the total cells. In MG-63 cells, ${ }^{*} \mathrm{P}<0.05$ compared with (MG-63) control and vector; in $\mathrm{U}-2$ cells, ${ }^{\prime} \mathrm{P}<0.05$ compared with (U-2) control and scramble. 
A

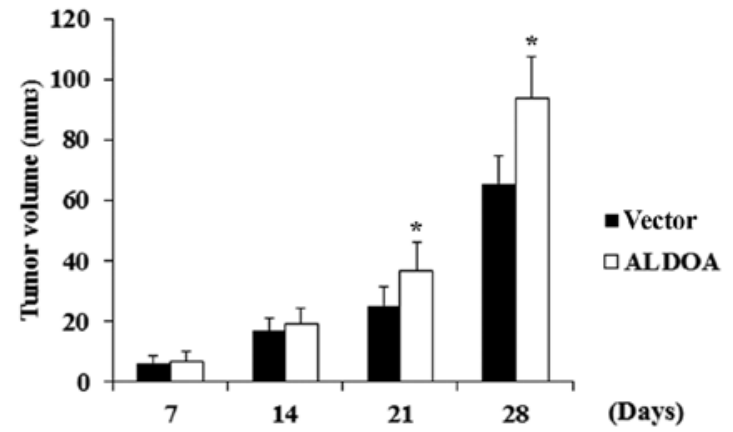

B

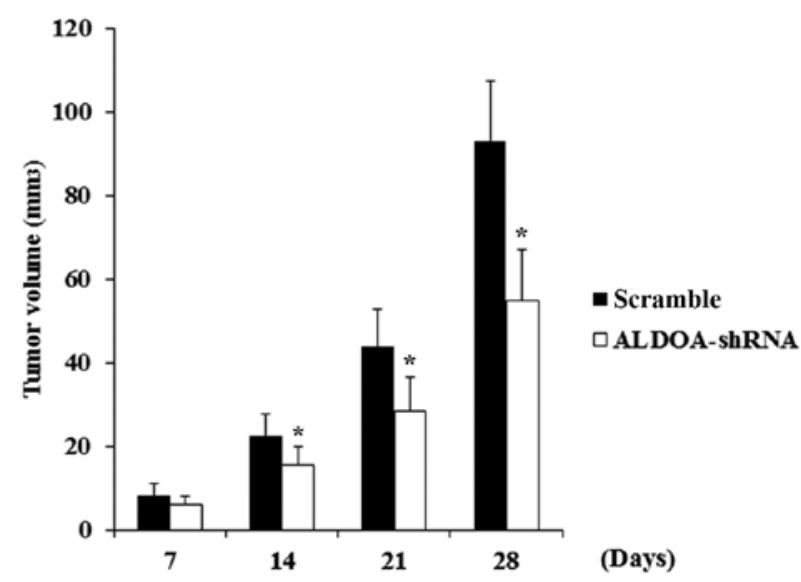

Figure 6. Change in tumor volume over time in the orthotopic xenograft osteosarcoma mouse model. (A) MG-63 cells stably transfected with empty pcDNA3 vector (vector) or ALDOA, and (B) U-2 cells stably transduced with scramble control shRNA (scramble) or ALDOA-shRNA were injected into the left tibia of individual nude mice, respectively. The primary tumor volume was monitored until 28 days post injection. In MG-63 cells, ${ }^{*} \mathrm{P}<0.05$ compared with vector; in U-2 cells, " $\mathrm{P}<0.05$ compared with scramble.

the MG-63 and U-2 cells under normal culture conditions (Fig. 5A). However, in the MG-63 cells treated with cisplatin, overexpression of ALDOA significantly decreased the rate of cell apoptosis when compared with the controls (Fig. 5B). In the U-2 cells, knockdown of ALDOA significantly increased cell apoptosis in the presence of cisplatin (Fig. 5C).

To assess the role of ALDOA in OS progression and metastasis in vivo, we employed an orthotopic xenograft OS nude mouse model. MG-63 cells stably transfected with the empty pcDNA3 expression vector or ALDOA, and U-2 cells stably transduced with scramble control shRNA or ALDOA-shRNA were used for intra-tibial injection, respectively. Twenty-eight days after the injection, $67 \%$ (6/9) of the mice in the vector control (vector) group, $100 \%$ (9/9) in the ALDOA overexpression group, $100 \%$ (9/9) in the scramble control (scramble) group, and $22 \%(2 / 9)$ in the ALDOA knockdown (ALDOA-shRNA) group exhibited obvious dyspnea and distress. All animals were sacrificed on day 28 post injection, and the lungs were collected. As shown in Fig. 6, mice injected with the MG-63 cells overexpressing ALDOA showed significantly larger primary tumors than those noted in the vector controls at day 21 post injection. In contrast, mice injected with the U-2 cells with ALDOA knockdown showed significantly smaller primary tumors than those noted in the scramble controls at day 14 post-injection. Compared with the vector control group, the
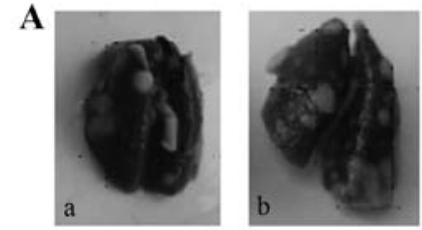

B

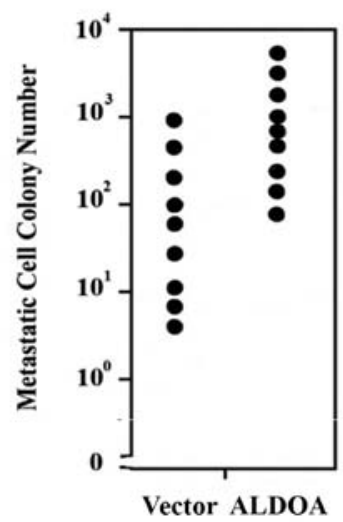

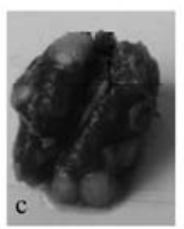

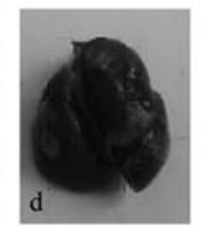

C

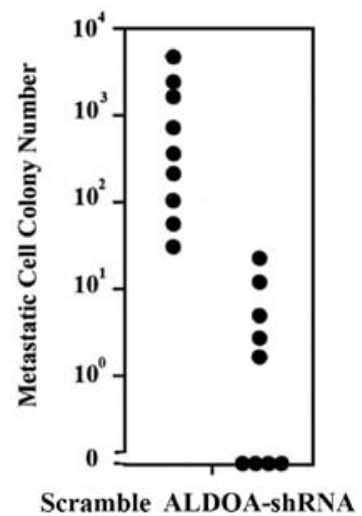

Figure 7. Pulmonary metastasis in the mouse model. (A) MG-63 cells stably transfected with empty pcDNA3 vector (vector) or ALDOA, and U-2 cells stably transduced with scramble control shRNA (scramble) or ALDOAshRNA were respectively used for tibial injection in mice. Typical lung images of mice injected with (a) vector, (b) ALDOA, (c) scramble and (d) ALDOA-shRNA are shown. Clonogenic lung metastasis assays were performed in mice injected with (B) MG-63 vector and ALDOA cells and (C) U-2 scramble and ALDOA-shRNA cells 28 days post injection. Each symbol represents a single animal ( $\mathrm{n}=9$ each group).

ALDOA overexpression group showed obviously more metastatic nodules on the lung surface as well as more swollen and congested lungs (Fig. 7A). In comparison with the scramble control group, the ALDOA knockdown group showed apparently fewer metastatic nodules on the lung surface as well as less swollen and congested lungs (Fig. 7A). To quantitate the pulmonary metastasis, clonogenic lung metastasis assays were performed. As shown in Fig. 7B, the number of lung metastases in the ALDOA overexpression group were significantly more than these numbers in the vector control group. In contrast, the ALDOA knockdown group showed markedly fewer metastases than the scramble control group (Fig. 7C).

To explore the effect of ALDOA on OS cell survival in vivo, we examined cell apoptosis in the primary tumors in the orthotopic xenograft OS mouse nude model. As shown in Fig. 8, mice injected with the MG-63 cells overexpressing ALDOA showed significantly lower OS cell apoptosis rates in the primary tumors than those injected with the vector control cells. On the other hand, mice injected with the U-2 cells with knockdown of ALDOA showed significantly higher OS cell apoptosis rates in the primary tumors than those injected with the scramble control cells.

\section{Discussion}

Inhibiting cancer cell glycolysis is an emerging therapeutic strategy for cancer (8). A recent study suggested that ALDOA, an important enzyme involved in glycolysis (8), is a negative survival marker of OS and may be implicated in OS 
A

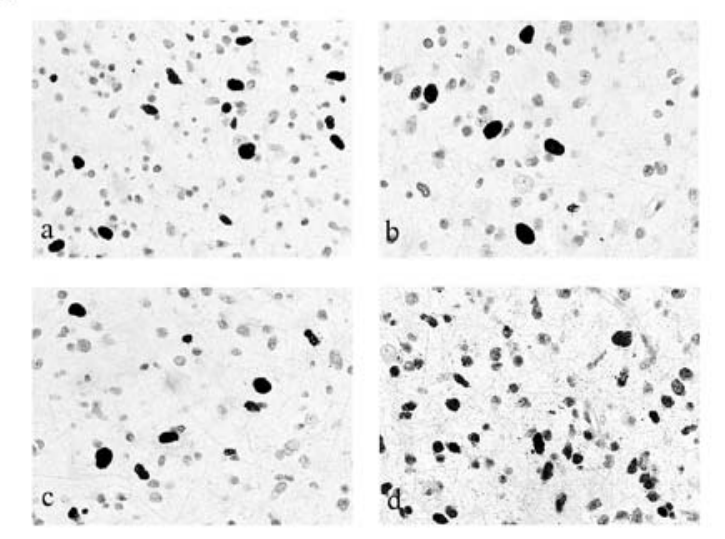

B

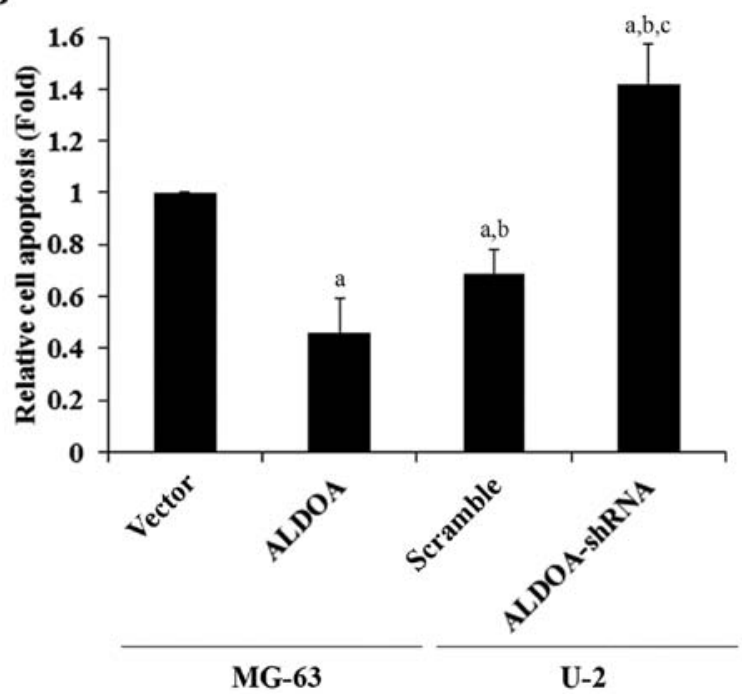

Figure 8. Cell apoptosis in the primary tumors in the orthotopic xenograft osteosarcoma mouse model. MG-63 cells stably transfected with empty pcDNA3 vector (vector) or ALDOA, and U-2 cells stably transduced with scramble control shRNA (scramble) or ALDOA-shRNA were respectively used for intra-tibia injection in mice. (A) Apoptosis was evaluated in the primary tumor tissue using the TUNEL (terminal deoxynucleotidyl transferase mediated nick-end labeling) technique 28 days post injection. Typical images of TUNEL staining of the primary tumor tissue derived from (a) vector, (b) ALDOA, (c) scramble (d) and ALDOA-shRNA cells are shown. All slides were counterstained with hematoxylin (magnification, $x 400$ ). (B) The cell apoptosis rate in the primary tumor tissue was expressed as fold-change to that of the (MG-63) vector control (designated as 1$)$. ${ }^{\mathrm{a}} \mathrm{P}<0.05$ compared with (MG-63) vector; ${ }^{b} \mathrm{P}<0.05$ compared with (MG-63) ALDOA; ${ }^{\mathrm{P}} \mathrm{P}<0.05$ compared with (U-2) scramble.

development and progression. In the present study, our in vitro data revealed that ALDOA promoted OS cell invasion and survival, and our in vivo data demonstrated an important role of ALDOA in promoting OS tumor growth and metastasis.

MG-63 and U-2 cells were used as OS cell models in the present study. MG-63 cells expressed a relatively low constitutive level of ALDOA when compared with the U-2 cells. Thus, overexpression and knockdown of ALDOA were respectively performed in the two cell lines to approach the study objectives from different angles. Changes in the aldolase activity were in line with those in the ALDOA expression levels, indicating that overexpression and knockdown of ALDOA indeed led to an alteration in the enzymatic activity in the OS cells.
OS is characterized by high local aggressiveness and a tendency to metastasize to the lungs, which remains a major cause of fatal outcome (4). Thus, we performed in vitro cell invasion assays to explore the effect of ALDOA on OS cell invasiveness. Overexpression of ALDOA increased cell invasion in MG-63 cells, which had a relatively low constitutive level of ALDOA expression and invasive activity, while knockdown of ALDOA nearly abolished cell invasion in the U-2 cells, which had a relatively high constitutive level of ALDOA expression and invasive activity. The findings suggest that ALDOA is critical for OS cell invasion. Among the different MMPs, MMP-2 showed expression level changes in line with the cell invasive activity changes in the OS cells, suggesting that ALDOA promotes OS cell invasion through upregulation of MMP-2 expression. Further studies are needed to ascertain how ALDOA regulates MMP-2 expression.

Cell survival against apoptotic stress is critical for cancer progression and metastasis (16). In the present study, we used a relatively small concentration of cisplatin $(10 \mathrm{nM})$ to induce apoptotic stress without killing most of the cells. In the presence of cisplatin, overexpression of ALDOA in the MG-63 cells significantly decreased cell apoptosis, while knockdown of ALDOA in the U-2 cells markedly increased cell apoptosis compared with the controls. The findings indicate that ALDOA is important for OS cell survival against apoptotic stress, which not only suggests a functional role for ALDOA in OS progression and metastasis, but also implicates ALDOA in the development of OS chemoresistance. Cisplatin elicits DNA repair mechanisms by crosslinking DNA, which in turn activates apoptosis when repair proves impossible (17). It is still unclear whether ALDOA impacts OS cell survival against other types of chemotherapeutic agents. Further studies with more types of chemotherapeutic agents and OS cell lines would elucidate this issue.

Based on the in vitro evidence that ALDOA plays an important role in OS cell invasion and survival, we used an orthotopic xenograft OS nude mouse model to further explore the functional role of ALDOA in OS progression and metastasis in vivo. As pulmonary metastasis is a major cause of fatal outcome in OS, we focused on lung metastasis in the mouse model, and the intra-tibial injection model in nude mice has proved to be a biologically relevant and adequate animal model for the induction of reproducible pulmonary metastasis (15). A combination of clinical signs, organ examinations, tumor volume analyses, and quantitative lung metastasis assays in the animal model demonstrated that ALDOA promotes OS primary tumor growth and pulmonary metastasis in vivo. Additionally, overexpression and knockdown of ALDOA decreased and increased cell apoptosis in the primary OS tumors, respectively, confirming the in vitro promoting effects of ALDOA on OS cell survival against the apoptotic stress induced by low-dose cisplatin.

The aldolase (ALDO) isozymes (A, B and C) are encoded by three different genes, differentially expressed during development. ALDOA is mainly produced by the developing embryo and in adult muscle; ALDOB is produced by liver, kidney and intestine; and ALDOC is mainly produced by brain and other nervous tissue. ALDOA and ALDOB have been associated with poor prognosis of OS and hepatocarcinomas, respectively $(10,18)$. Future studies of whether and how 
ALDOB and ALDOC are involved in OS development and progression are warranted.

In conclusion, the present study provides the first in vitro and in vivo evidence supporting a critical functional role of ALDOA in OS progression and metastasis, suggesting that ALDOA could serve as a novel therapeutic target in OS. Additionally, our results also suggest that ALDOA is involved in the development of OS chemoresistance.

\section{References}

1. Ottaviani G and Jaffe N: The epidemiology of osteosarcoma. Cancer Treat Res 152: 3-13, 2010.

2. Bacci G, Briccoli A, Rocca M, et al: Neoadjuvant chemotherapy for OS of the extremities with metastases at presentation: recent experience at the Rizzoli Institute in 57 patients treated with cisplatin, doxorubicin, and a high dose of methotrexate and ifosfamide. Ann Oncol 14: 1126-1134, 2003.

3. Kager L, Zoubek A, Potschger U, et al: Primary metastatic OS presentation and outcome of patients treated on neoadjuvant Cooperative OS Study Group protocols. J Clin Oncol 21: 2011-2018, 2003.

4. Ta HT, Dass CR, Choong PF and Dunstan DE: Osteosarcoma treatment: state of the art. Cancer Metastasis Rev 28: 247-263, 2009.

5. Gorlick R, Anderson P and Andrulis I: Biology of childhood osteogenic sarcoma and potential targets for therapeutic development: meeting summary. Clin Cancer Res 9: 5442-5453, 2003.

6. Wittig JC, Bickels J and Priebat D: Osteosarcoma: a multidisciplinary approach to diagnosis and treatment. Am Fam Physician 65: 1123-1132, 2002.

7. Gatenby RA and Gillies RJ: Why do cancers have high aerobic glycolysis? Nat Rev Cancer 4: 891-899, 2004.

8. Scatena R, Bottoni P, Pontoglio A, Mastrototaro L and Giardina B: Glycolytic enzyme inhibitors in cancer treatment. Expert Opin Investig Drugs 17: 1533-1545, 2008.
9. Marin-Hernandez A, Rodríguez-Enríquez S, Vital-González PA, et al: Determining and understanding the control of glycolysis in fast-growth tumor cells. Flux control by an over-expressed but strongly product-inhibited hexokinase. FEBS J 273: 1975-1988, 2006 .

10. Chen X, Yang TT, Zhou Y, et al: Proteomic profiling of osteosarcoma cells identifies aldoa and sult1a3 as negative survival markers of human osteosarcoma. Mol Carcinog: Sep 4, 2012, (Epub ahead of print).

11. Sakakibara M, Takahashi I, Takasaki Y, Mukai T and Hori K: Construction and expression of human aldolase A and B expression plasmids in Escherichia coli host. Biochim Biophys Acta 1007: 334-342, 1989.

12. Dass CR, Ek ET, Contreras KG and Choong PF: A novel orthotopic murine model provides insights into cellular and molecular characteristics contributing to human osteosarcoma. Clin Exp Metastasis 23: 367-380, 2006.

13. Ek ETH, Dass CR, Contreras KG and Choong PFM: Inhibition of orthotopic osteosarcoma growth and metastasis by multitargeted antitumour activities of pigment epithelium-derived factor. Clin Exp Metastasis 24: 93-106, 2007.

14. Pilones KA, Kawashima N, Yang AM, et al: Invariant natural killer $\mathrm{T}$ cells regulate breast cancer response to radiation and CTLA-4 blockade. Clin Cancer Res 15: 597-606, 2009.

15. Li Y, Liao Q, Li K, et al: Knockdown of endothelin A receptor expression inhibits osteosarcoma pulmonary metastasis in an orthotopic xenograft mouse model. Mol Med Rep 5: 1391-1395, 2012.

16. Hopkin K, Edwards P, Harris A, Klausner R, Peters G, Selby P and Stanley M: Cancer. In: Molecular Biology of the Cell. Alberts B, Johnson A and Lewis J (eds). 4th edition. Garland Science, New York, pp1324-1325, 2002.

17. Rosenberg B, Vancamp L, Trosko JE and Mansour VH: Platinum compounds: a new class of potent antitumour agents. Nature 222: 385-386, 1969.

18. Peng SY, Lai PL, Pan HW, Hsiao LP and Hsu HC: Aberrant expression of the glycolytic enzymes aldolase B and type II hexokinase in hepatocellular carcinoma are predictive markers for advanced stage, early recurrence and poor prognosis. Oncol Rep 19: 1045-1053, 2008 . 